\title{
Efeito do processamento industrial na qualidade e na segurança de salgados prontos para comer
}

\author{
Effects of industrial processing on the quality and safety of ready-to-eat savouries
}

\author{
Tânia Gonçalves Albuquerque ${ }^{1,2}$, Mafalda Alexandra Silva', Maria Beatriz Prior Pinto Oliveira ${ }^{2}$, Helena Soares Costa ${ }^{1,2 \star}$ \\ 1 Instituto Nacional de Saúde Doutor Ricardo Jorge, Lisboa - Portugal \\ 2 Universidade do Porto, Faculdade de Farmácia, Laboratório Associado para a Química Verde - REQUIMTE-LAQV, Lisboa - Portugal
}

\begin{abstract}
*Corresponding Author
Helena Soares Costa, Instituto Nacional de Saúde Doutor Ricardo Jorge, Avenida Padre Cruz, CEP: 1649-016, Lisboa - Portugal, e-mail: helena.costa@insa.min-saude.pt
\end{abstract}

Cite as: Effects of industrial processing on the quality and safety of ready-to-eat savouries. Braz. J. Food Technol., v. 20, e2017012, 2017.

Received: Feb. 14, 2017; Accepted: Apr. 11, 2017

\section{Resumo}

O estilo de vida das famílias tem sofrido alterações profundas, traduzindo-se, entre outros fatores, na redução do tempo para preparação das refeições. Pelo referido, as vendas de alimentos confeccionados, caso dos salgados, têm tido um crescimento contínuo. Este trabalho de investigação teve como objetivos: (1) determinar os teores de sal, gordura total e o perfil em ácidos graxos de salgados prontos para comer; (2) correlacionar os diversos componentes analisados; (3) analisar a evolução da composição dos salgados com base na comparação com os valores da literatura, e (4) estimar os benefícios/riscos para a saúde da população, tendo por base as recomendações de referência. Foram avaliados, em 2015, 12 tipos de salgados prontos para comer. O teor de gordura total foi determinado pelo método de hidrólise ácida, seguido de extração em Soxhlet, com éter de petróleo. O teor de sal foi determinado utilizando-se o método de Charpentier-Volhard. Para o perfil de ácidos graxos, utilizou-se um método de cromatografia gasosa com detecção por ionização em chama. O teor de gordura variou entre $9,14 \mathrm{~g} / 100 \mathrm{~g}$ (pataniscas de bacalhau) e 28,9 g/100 g (chamuças de carne). O teor de sal mais elevado foi de $2,13 \mathrm{~g} / 100 \mathrm{~g}$ (croquetes de carne). No entanto, uma unidade de empada de requeijão pode contribuir com $45 \%$ da ingestão diária de sal recomendada. Os ácidos graxos majoritários, em 58\% das amostras analisadas, eram poli-insaturados, e os seus teores variaram entre 3,54 g/100 g (empadas de galinha) e 15,5 g/100 g (chamuças de carne). Todas as amostras analisadas tinham teores de ácidos graxos trans inferiores a $2 \%$ do teor de gordura total. De uma forma geral, os salgados prontos para comer analisados tinham teores elevados de gordura e de sal, devendo, por isso, o seu consumo ser moderado. No entanto, na maior parte das amostras, o teor de ácidos graxos insaturados era superior aos saturados. No que se refere aos teores de ácidos graxos trans, estes alimentos podem ser considerados seguros.

Palavras-chave: Ácidos graxos trans; Gordura saturada; Qualidade nutricional; Sal; Salgados prontos para comer.

\section{Abstract}

Family lifestyle has changed greatly over the years, and nowadays there is a lack of time to prepare meals amongst other factors. Hence the sales of ready-to-eat foods, such as savouries, have shown continuous growth. The aim of this study was: (1) to determine the salt and total fat contents and the fatty acid profile of ready-to-eat savouries; (2) to correlate the different components analysed; (3) to analyse the evolution of the composition of savouries based on a comparison with the values found in the literature; and (4) to estimate the health benefits/risks to the population based on the reference dietary recommendations. In 2015, 12 types of ready-to-eat savouries were evaluated. The total fat content was determined by the acid hydrolysis method, followed by the Soxhlet extraction with petroleum ether. The salt content was determined using the Charpentier-Volhard method. Gas chromatography with flame ionization detection was used to determine the fatty acid profile. The fat content varied between $9.14 \mathrm{~g} / 100 \mathrm{~g}$ (a cod savoury) and $28.9 \mathrm{~g} / 100 \mathrm{~g}$ (a meat savoury). The highest salt content was $2.13 \mathrm{~g} / 100 \mathrm{~g}$ (meat croquettes). However, one unit of curd cheese pie can contribute $45 \%$ of the recommended daily intake of salt. In $58 \%$ of the samples analysed, the major fatty acids were polyunsaturated, and the levels of these fatty acids varied between $3.54 \mathrm{~g} / 100 \mathrm{~g}$ (chicken pies) and $15.5 \mathrm{~g} / 100 \mathrm{~g}$ (meat savouries). All the samples analysed had trans 
fatty acid contents below $2 \%$ of the total fat content. In general, the ready-to-eat savouries analysed showed high levels of fat and salt, and should therefore be consumed in moderation. However, in the majority of the samples the unsaturated fatty acid content was higher than the saturated fatty acid content. With respect to the levels of trans fatty acids, these foods could be considered safe.

Keywords: trans fatty acids; Saturated fat; Nutritional quality; Salt; Ready-to-eat savouries.

\section{Introdução}

Os avanços tecnológicos, as mudanças nas estruturas domésticas, as sociedades multiculturais, bem como a mudança dos valores sociais, resultaram num aumento da procura por alimentos que sejam de preparação rápida e fácil (BOER et al., 2004; SANTOS; OLIVEIRA, 2012). O estilo de vida das famílias tem sofrido alterações profundas, traduzindo-se essencialmente na falta de tempo para adquirir os alimentos e preparar as refeições. Por esse motivo, adquirem cada vez mais alimentos confeccionados ou pré-confeccionados, também conhecidos por alimentos de conveniência (ALBUQUERQUE et al., 2012, 2016a). No entanto, hoje em dia, o consumidor está, também, cada vez mais atento, consciente e preocupado com a sua saúde, exigindo alimentos nutricionalmente adequados e seguros, e reconhece que alguns destes alimentos são ricos em sal e gordura (BERBARI et al., 2011).

O consumo excessivo de alimentos com elevados teores de sal está relacionado com um maior risco de desenvolvimento de hipertensão arterial (IGNÁCIO et al., 2013). Neste sentido, têm sido desenvolvidas diversas iniciativas, um pouco por todo o mundo, visando reduzir as quantidades de sal presentes nos alimentos (WHO, 2013). Em Portugal, até a data da realização deste trabalho, apenas estava legislado um valor máximo para o teor de sal no pão (POLÓNIA et al., 2014; SPH, 2016). Apesar disso, esta medida tem tido êxito em Portugal, uma vez que o consumo de sal decresceu de 12,3 para $10,7 \mathrm{~g} / \mathrm{dia}$, e pretende-se continuar a implementar medidas legislativas no sentido de fixar limites para outros alimentos, sobretudo os alimentos processados (POLÓNIA et al., 2014; SPH, 2016).

Relativamente à gordura, nos últimos anos, tem-se verificado que os trabalhos de investigação e os guias de recomendações internacionais dão cada vez mais importância ao tipo de gordura presente nos alimentos, nomeadamente a gordura saturada e a trans, e não só à quantidade de gordura total. A União Europeia, nos últimos cinco anos, tem desenvolvido diversas iniciativas no sentido de avaliar o teor de ácidos graxos trans (AGT) nos alimentos, dado que, em nível Europeu, ainda não existe uma medida legislativa que fixe o limite máximo para os alimentos, nem que obrigue a sua declaração na rotulagem (WHO, 2015; UNIÃO EUROPEIA, 2011). No entanto, noutros países, como, por exemplo, o Brasil, é obrigatória a declaração dos níveis de gordura trans nos rótulos dos alimentos industrializados, quando o teor é superior a 0,2 g/porção (PINTO et al., 2016). Uma ingestão elevada destes ácidos graxos tem sido relacionada com um aumento do risco de desenvolvimento de doenças crônicas, nomeadamente as do foro cardiovascular (MOZAFFARIAN et al., 2006).

Ao longo dos últimos anos, tem-se observado uma maior oferta/disponibilidade de alimentos de conveniência, como, por exemplo, os salgados prontos para comer, estando estes acessíveis em cafés, pastelarias, bares, restaurantes, supermercados, grandes superfícies, entre outros. Os salgados são usados como um "snack" ou como um elemento da refeição principal. Também em relação ao modo de preparação, têm surgido no mercado novas alternativas e, atualmente, além de ser possível continuar a utilizar a fritura, também já é possível encontrar alguns salgados que podem ser adquiridos na forma pré-frita e serem, posteriormente, preparados no forno (ALBUQUERQUE et al., 2016a).

No entanto, a fritura continua a ser o método mais usado para a preparação industrial e doméstica de salgados prontos para comer, não só porque é um método de confecção extremamente rápido, mas também por conferir aos alimentos propriedades organoléticas únicas, como o sabor, o aroma e a textura (DAMY; JORGE, 2003; MALACRIDA; JORGE, 2003). Todavia, a utilização desta técnica é muitas vezes questionada, pelo fato de ocorrerem inúmeras reações químicas que colocam em causa a qualidade e a segurança, quer do óleo de fritura, quer do alimento frito (DAMY; JORGE, 2003; MALACRIDA; JORGE, 2003). Alguns dos fatores identificados como responsáveis por estas alterações são o uso prolongado dos óleos e a utilização de temperaturas elevadas.

Neste trabalho, determinaram-se os teores de sal e de gordura total, e o perfil em ácidos graxos de salgados prontos para comer. Discutem-se os resultados obtidos e correlacionam-se os diversos componentes determinados, analisa-se a evolução da composição dos salgados com base nos valores da literatura e estimam-se os benefícios/riscos do seu consumo, tendo por base as recomendações de referência.

\section{Material e métodos}

\subsection{Amostragem}

Foram adquiridos, em 2015, em diversas regiões de Lisboa, 12 tipos de salgados prontos para comer: rolinhos de salsicha, rissóis de camarão, croquetes de 
Efeito do processamento industrial na qualidade e na segurança de salgados prontos para comer

Albuquerque, T. G. et al.

carne, chamuças de carne, coxas de frango, pastéis de bacalhau, crepes de legumes, pataniscas de bacalhau, empanadas de atum, empadas de requeijão, empadas de galinha e rissóis de leitão (Figura 1). A seleção das amostras foi realizada com base num estudo de mercado prévio, que avaliou os tipos de produtos disponibilizados ao consumidor nas várias grandes superfícies da região de Lisboa (Portugal). De acordo com a informação fornecida pelos distribuidores, estes produtos foram confeccionados no forno (empanadas de atum, empadas de requeijão e empadas de galinha) ou sujeitos à fritura por imersão, não tendo sido possível obter informação relativa à gordura empregada ou ao óleo utilizado. Para cada tipo de salgado, foram recolhidas diversas unidades, em diferentes grandes superfícies. Após recepção das amostras no laboratório, foram registradas todas as informações consideradas relevantes e as amostras foram homogeneizadas utilizando-se um triturador (GRINDOMIX ${ }^{\circledR}$, GM200, Alemanha) durante aproximadamente $1 \mathrm{~min}$ a $5.000 \mathrm{rpm}$. Em seguida, as amostras foram acondicionadas em frascos de plástico com tampa, mantidas ao abrigo da luz e do calor, e conservadas numa arca frigorífica a $4{ }^{\circ} \mathrm{C}$. As análises foram realizadas em triplicado para todos os parâmetros.

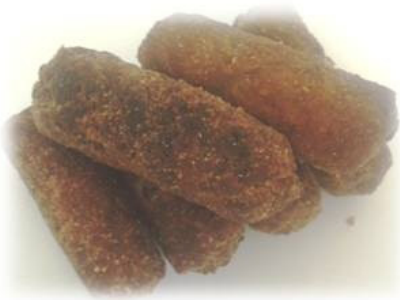

Croquetes

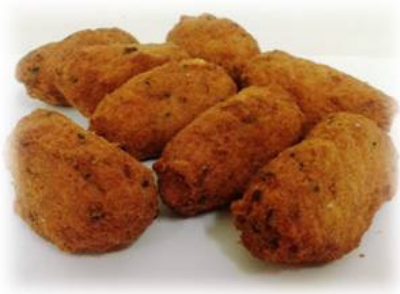

Pastéis de bacalhau

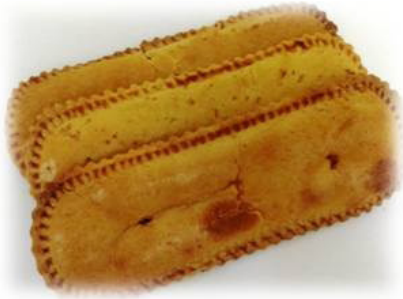

Empanadas de atum

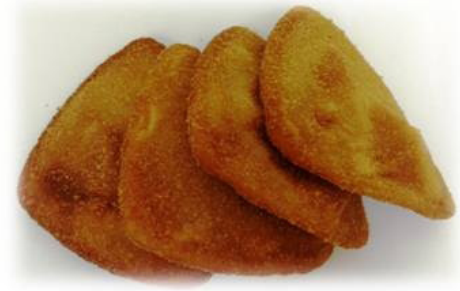

Rissóis de leitão

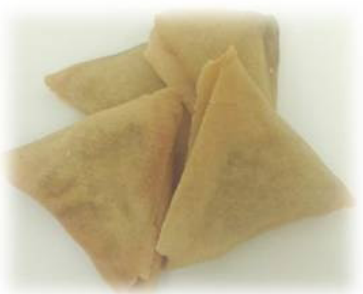

Chamuças de carne

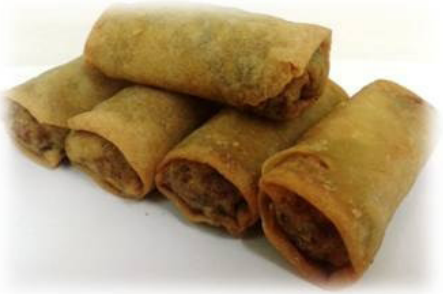

Crepes de legumes

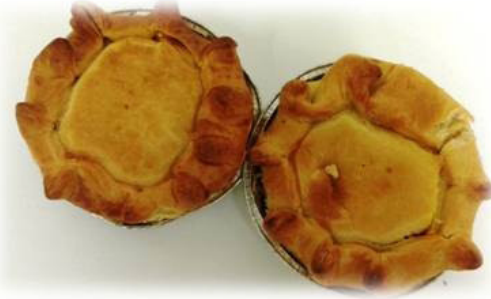

Empadas de

requeijão

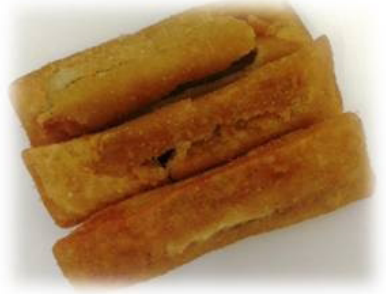

Rolinhos de

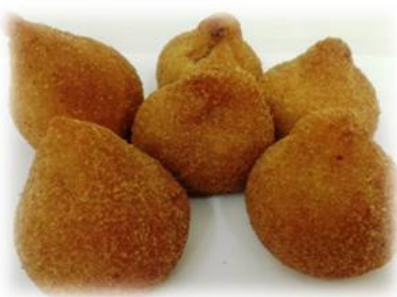

Coxas de frango

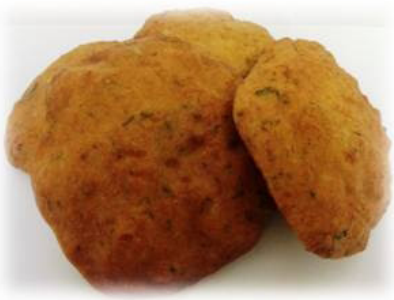

Pataniscas de bacalhau

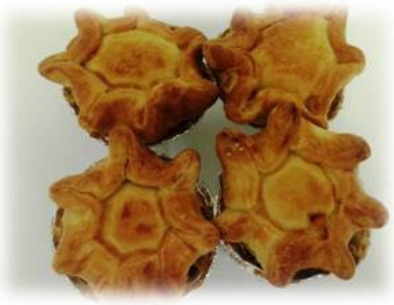

Empadas de
galinha

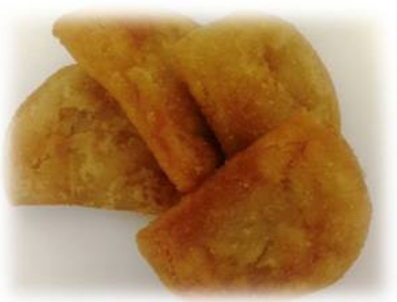

Rissóis de camarão

Figura 1. Exemplos das amostras de salgados prontos para comer analisadas. 


\subsection{Determinação do teor de sal}

O teor de sal foi determinado pelo método de Charpentier-Volhard (ALBUQUERQUE et al., 2012). Este método baseia-se na extração dos cloretos em água ultrapura, seguida de precipitação dos cloretos pelo nitrato de prata, e, por último, a titulação do excesso de nitrato de prata com tiocianato de potássio.

\subsection{Determinação do teor de gordura total}

O teor de gordura total foi determinado pelo método de hidrólise, com uma solução de ácido clorídrico, seguindo-se uma filtração; posteriormente, procedeu-se à secagem da matéria gorda retida no filtro e à extração em Soxhlet (Soxtec ${ }^{\text {TM }}$ 2050, Auto Fat Extraction System, FOSS Analytical, Hilleroed, Dinamarca), com éter de petróleo. Por fim, a eliminação do solvente por evaporação, a secagem e a pesagem do extrato (ALBUQUERQUE et al., 2016b).

\subsection{Determinação do perfil de ácidos graxos}

Para a determinação do perfil de ácidos graxos, foi realizada uma extração de gordura utilizando-se éter de petróleo (ALBUQUERQUE et al., 2016a). Posteriormente, realizou-se uma transesterificação a frio, com hidróxido de potássio metanólico e n-heptano. Para a análise por cromatografia gasosa acoplada à detecção por ionização em chama, utilizou-se uma coluna capilar Supelco ${ }^{\mathrm{TM}}$ 2560 (100 m × 0,25 mm d.i.; 0,2 4 m espessura do filme, Supelco ${ }^{\text {TM }}$, CA, USA). Foi utilizado como gás de arrasto o hélio. Injetou-se $1 \mu \mathrm{L}$ de cada amostra a analisar em modo split 50:1. As temperaturas do injetor e do detector foram mantidas a $240^{\circ} \mathrm{C}$. Foi utilizada a seguinte rampa de temperatura: $60^{\circ} \mathrm{C}(1 \mathrm{~min})$, aumentar até $168^{\circ} \mathrm{C}$ a $17^{\circ} \mathrm{C} / \mathrm{min}$ (manter $28 \mathrm{~min}$ ) e, em seguida, aumentar até $235^{\circ} \mathrm{C}$ a $4{ }^{\circ} \mathrm{C} / \mathrm{min}$ (manter $15 \mathrm{~min}$ ).

\subsection{Análise estatística}

Todos os dados foram analisados com os programas Statistica 6413.0 software (Statsoft Inc., Tulsa, EUA) e Microsoft Office Excel ${ }^{\circledR}$ 2010. Foram efetuados também testes de correlação de Spearman e as médias foram comparadas utilizando-se o teste de Tukey. As diferenças observadas foram consideradas significativas quando o valor de $p<0,05$. Os resultados estão expressos em g/100 g de amostra e apresentados na forma de média \pm desvio padrão $(n=3)$ ou percentagem, utilizando-se algarismos significativos.

\section{Resultados e discussão}

\subsection{Teor de sal e de gordura total}

Os resultados relativos aos teores de gordura total e sal (por $100 \mathrm{~g}$ ) das amostras de salgados prontos para comer apresentam-se na Tabela 1. O teor de gordura total variou entre 9,1 $\pm 0,1 \mathrm{~g} / 100 \mathrm{~g}$ e 28,9 \pm 0,04 g/100 g para as pataniscas de bacalhau e as chamuças de carne, respectivamente (Tabela 1).

Em Portugal, até a atual data, existe pouca informação sobre a composição nutricional dos salgados prontos para comer. Tanto quanto é do conhecimento dos autores, apenas na Tabela da Composição de Alimentos (TCA) é possível encontrar alguma informação relativa a amostras semelhantes (PORTUGAL, 2015). Para efeitos de comparação entre os valores determinados no presente trabalho e a TCA, com a finalidade de fazer um ponto de situação relativo à evolução dos teores de gordura, sal, ácidos graxos saturados (AGS) e AGT, nos últimos anos,

Tabela 1. Teores de sal, gordura total, ácidos graxos saturados, monoinsaturados, poli-insaturados e trans (por $100 \mathrm{~g}$ ) dos salgados prontos para comer analisados.

\begin{tabular}{|c|c|c|c|c|c|c|}
\hline Amostras & $\begin{array}{l}\text { Sal } \\
\text { (g) }\end{array}$ & $\begin{array}{c}\text { Gordura total } \\
\text { (g) }\end{array}$ & $\begin{array}{l}\text { AGS } \\
\text { (g) }\end{array}$ & $\begin{array}{c}\text { AGMI } \\
\text { (g) }\end{array}$ & $\begin{array}{l}\text { AGPI } \\
\text { (g) }\end{array}$ & $\begin{array}{l}\text { AGT } \\
\text { (mg) }\end{array}$ \\
\hline Rolinhos de salsicha & $1,67 \pm 0,1^{g}$ & $13,2 \pm 0,02 d$ & $2,43 \pm 0,01^{e}$ & $4,05 \pm 0,1^{d}$ & $6,04 \pm 0,04^{n}$ & $13,2 \pm 0,22$ \\
\hline Rissóis de camarão & $1,91 \pm 0,2^{h}$ & $20,1 \pm 0,3$ & $2,36 \pm 0,01^{d}$ & $5,72 \pm 0,02^{h}$ & $11,0 \pm 0,1^{k}$ & $9,93 \pm 0,13 b$ \\
\hline Croquetes de carne & $2,13 \pm 0,1^{i}$ & $15,5 \pm 0,3^{g}$ & $3,15 \pm 0,002^{g}$ & $4,94 \pm 0,001^{f}$ & $6,59 \pm 0,02$ & $15,2 \pm 0,16^{\circ}$ \\
\hline Chamuças de carne & $1,32 \pm 0,02 \mathrm{e}$ & $28,9 \pm 0,04^{k}$ & $3,75 \pm 0,001^{h}$ & $8,28 \pm 0,2^{\prime}$ & $15,5 \pm 0,3^{\prime}$ & $14,9 \pm 0,38$ \\
\hline Coxas de frango & $1,29 \pm 0,01^{\mathrm{e}}$ & $10,3 \pm 0,4^{b}$ & $2,36 \pm 0,001^{d}$ & $3,73 \pm 0,01^{c}$ & $3,67 \pm 0,01^{b}$ & $18,8 \pm 0,21^{e}$ \\
\hline Pastéis de bacalhau & $1,01 \pm 0,02^{c}$ & $11,7 \pm 0,6^{c}$ & $2,04 \pm 0,04^{c}$ & $5,33 \pm 0,039$ & $3,73 \pm 0,02^{\circ}$ & $9,96 \pm 0,08 b$ \\
\hline Crepes de legumes & $0,762 \pm 0,1^{a}$ & $14,1 \pm 0,1^{f}$ & $2,36 \pm 0,001^{d}$ & $6,23 \pm 0,1^{i}$ & $4,85 \pm 0,001^{f}$ & $13,4 \pm 0,08$ \\
\hline Pataniscas de bacalhau & $1,50 \pm 0,05^{f}$ & $9,14 \pm 0,1^{a}$ & $1,34 \pm 0,01^{a}$ & $2,94 \pm 0,01^{a}$ & $4,41 \pm 0,02 e^{e}$ & $7,76 \pm 0,13$ a \\
\hline Empanadas de atum & $1,55 \pm 0,04^{f}$ & $14,2 \pm 0,1^{f}$ & $1,81 \pm 0,004 b$ & $3,68 \pm 0,002^{b}$ & $8,00 \pm 0,1^{i}$ & $13,2 \pm 0,72^{\circ}$ \\
\hline Empadas de requeijão & $1,16 \pm 0,1^{d}$ & $18,3 \pm 0,2^{h}$ & $6,61 \pm 0,003^{i}$ & $6,37 \pm 0,01^{i}$ & $4,24 \pm 0,02^{d}$ & $105 \pm 0,629$ \\
\hline Empadas de galinha & $0,877 \pm 0,02 b$ & $19,2 \pm 0,6$ & $6,99 \pm 0,003$ & $7,65 \pm 0,1^{k}$ & $3,54 \pm 0,01^{a}$ & $54,3 \pm 0,19$ \\
\hline Rissóis de leitão & $0,776 \pm 0,02^{a}$ & $13,4 \pm 0,1^{\mathrm{e}}$ & $2,56 \pm 0,002^{f}$ & $4,33 \pm 0,01^{\mathrm{e}}$ & $5,82 \pm 0,02^{g}$ & $15,5 \pm 0,02$ \\
\hline
\end{tabular}

Os resultados estão apresentados na forma de média \pm desvio padrão ( $n=3)$; AGS: Ácidos Graxos Saturados; AGMI: Ácidos Graxos Monoinsaturados; AGPI: Ácidos Graxos Poli-insaturados; AGT: Ácidos Graxos trans. Valores na mesma coluna com letras diferentes (a-l) apresentam diferenças significativas $(p<0,05)$. 
apenas foi possível utilizar cinco das amostras. No que diz respeito ao teor de gordura, verificou-se que três das amostras analisadas neste estudo apresentaram teores de gordura inferiores, comparativamente aos valores apresentados na TCA (PORTUGAL, 2015). No entanto, a diferença mais considerável refere-se aos rissóis de camarão, apresentando, os salgados desse tipo analisados neste estudo, teores superiores (aproximadamente $10 \mathrm{~g}$ ) aos descritos na TCA.

O Regulamento (UE) n. ${ }^{\circ} 1169 / 2011$ de 25 de outubro de 2011, relativo à prestação de informação aos consumidores sobre os gêneros alimentícios, determina que a dose de referência para o teor de gordura é de $70 \mathrm{~g} / \mathrm{dia}$, tendo por base uma dieta padrão de 8.400 kJ/2.000 kcal, para um adulto (UNIÃO EUROPEIA, 2011). O contributo (\%) de uma unidade de salgados varia entre 7 e 50\% da dose de referência de gordura diária, respectivamente para os pastéis de bacalhau e as empadas de requeijão (Tabela 2).

Foi também realizada uma análise comparativa dos salgados confeccionados no forno (empanadas de atum, empadas de requeijão e empadas de galinha) e os produtos sujeitos à fritura, para avaliar se, em termos médios, existia diferença entre os teores de gordura. Verificou-se que a média dos teores de gordura dos alimentos confeccionados no forno é superior (17,2 g/100 g vs. 15,1 g/100 g), mas não se verificaram diferenças significativas.

O teor de sal mais elevado foi de 2,1 $\pm 0,1 \mathrm{~g} / 100 \mathrm{~g}$ (croquetes de carne). Comparando-se os resultados obtidos com os da TCA, verificou-se que as diferenças não são tão evidentes, como no caso do teor de gordura. As diferenças devem-se, sobretudo, à adição de sal durante a preparação dos salgados. A ingestão excessiva de alimentos ricos em sal está diretamente relacionada com o risco de desenvolvimento de hipertensão arterial. Os alimentos processados são reconhecidos como alguns dos que mais contribuem para esta ingestão. A Organização Mundial de Saúde recomenda uma ingestão de sal inferior a 5 g/dia, para prevenir doenças cardiovasculares (WHO, 2013). Se considerarmos os resultados obtidos, verifica-se que, por unidade, uma empada de requeijão pode contribuir com $45 \%$ do valor recomendado para a ingestão diária de sal, podendo constituir um fator de preocupação para a saúde da população portuguesa.

\subsection{Composição em ácidos graxos}

O teor de AGS variou entre 1,34 g/100 g e 6,99 g/100 g, para as pataniscas de bacalhau e as empadas de galinha, respectivamente (Tabela 1). Uma elevada ingestão deste tipo de ácidos graxos tem sido relacionada com um aumento do risco de desenvolvimento de doenças cardiovasculares. No entanto, os AGS podem ter efeitos distintos nas concentrações plasmáticas de colesterol. De acordo com a literatura, os ácidos láurico (C12:0), mirístico (C14:0) e palmítico (C16:0) contribuem para o aumento do colesterol das lipoproteínas de baixa densidade, enquanto o ácido esteárico (C18:0) não demonstrou qualquer efeito (EFSA, 2010). Note-se que apenas as empadas de requeijão apresentaram os ácidos graxos saturados como majoritários.

De acordo com o Regulamento (UE) n. ${ }^{\circ} 1169 / 2011$, a dose de referência para os AGS é de $20 \mathrm{~g} /$ dia (UNIÃO EUROPEIA, 2011). Nesse sentido, foi realizada uma análise do contributo de uma unidade de cada tipo dos salgados analisados para a ingestão de AGS. Os valores médios das unidades de salgados analisados estão apresentados na Tabela 2. Os salgados (por unidade) que contribuem para uma maior ingestão de AGS são a empada de

Tabela 2. Composição (g) em gordura total, sal e ácidos graxos saturados dos salgados prontos para comer analisados e contributo para a dose de referência.

\begin{tabular}{|c|c|c|c|c|c|c|c|}
\hline \multirow{2}{*}{ Amostras } & \multirow{2}{*}{$\begin{array}{l}\text { Unidade } \\
\text { (g) }\end{array}$} & \multicolumn{2}{|c|}{$\begin{array}{l}\text { Sal } \\
(g)\end{array}$} & \multicolumn{2}{|c|}{$\begin{array}{c}\text { Gordura } \\
\text { (g) }\end{array}$} & \multicolumn{2}{|c|}{$\begin{array}{c}\text { Ácidos graxos saturados } \\
\text { (g) }\end{array}$} \\
\hline & & $\begin{array}{c}\text { Por } \\
\text { unidade }\end{array}$ & $\begin{array}{c}\text { Contributo } \\
(\%)\end{array}$ & $\begin{array}{c}\text { Por } \\
\text { unidade }\end{array}$ & $\begin{array}{c}\text { Contributo } \\
(\%)\end{array}$ & $\begin{array}{c}\text { Por } \\
\text { unidade }\end{array}$ & $\begin{array}{c}\text { Contributo } \\
(\%)\end{array}$ \\
\hline Rolinhos de salsicha & 80 & 1,33 & 27 & 10,5 & 15 & 1,94 & 10 \\
\hline Rissóis de camarão & 45 & 0,862 & 17 & 9,03 & 13 & 1,06 & 5 \\
\hline Croquetes de carne & 37 & 0,784 & 16 & 5,70 & 8 & 1,16 & 6 \\
\hline Chamuças de carne & 49 & 0,649 & 13 & 14,2 & 20 & 1,84 & 9 \\
\hline Coxas de frango & 67 & 0,859 & 17 & 6,86 & 10 & 1,57 & 8 \\
\hline Pastéis de bacalhau & 45 & 0,449 & 9 & 5,21 & 7 & 0,910 & 5 \\
\hline Crepes de legumes & 62 & 0,474 & 9 & 8,78 & 13 & 1,47 & 7 \\
\hline Pataniscas de bacalhau & 80 & 1,20 & 24 & 7,30 & 10 & 1,07 & 5 \\
\hline Empanadas de atum & 125 & 1,94 & 39 & 17,8 & 25 & 2,27 & 11 \\
\hline Empadas de requeijão & 193 & 2,24 & 45 & 35,3 & 50 & 12,8 & 64 \\
\hline Empadas de galinha & 92 & 0,807 & 16 & 17,7 & 25 & 6,44 & 32 \\
\hline Rissóis de leitão & 91 & 0,706 & 14 & 12,2 & 17 & 2,33 & 12 \\
\hline
\end{tabular}


requeijão (64\%) e a empada de galinha (32\%), sendo a dose de referência facilmente ultrapassável em um dia, ou seja, alimentar-se além do limite diário definido para AGS não é difícil, quando se ingerem salgados prontos para comer, como a empada de requeijão e a empada de galinha (Tabela 2).

De forma geral, as amostras analisadas apresentaram majoritariamente ácidos graxos insaturados. Está cientificamente provado que a substituição dos AGS por ácidos graxos insaturados é benéfica para a saúde e diminui o risco de desenvolvimento de algumas doenças crônicas (EFSA, 2010). No caso específico dos salgados prontos para comer, a presença de ácidos graxos insaturados está, em grande parte, relacionada com a qualidade/quantidade de gordura absorvida durante o processo de fritura. Hoje em dia, cada vez mais as gorduras de origem saturada, nomeadamente banha, manteiga e gordura de palma, são substituídas por óleos vegetais ricos em ácidos graxos insaturados, nomeadamente óleos de girassol, soja e colza, ou misturas de óleos. Estas reformulações e modificações introduzidas pela indústria alimentar podem trazer benefícios à qualidade nutricional dos alimentos, que se traduzem num impacto menos negativo na saúde da população que os consome. Neste trabalho, o teor de ácidos graxos monoinsaturados (AGMI) variou entre 2,94 g/100 g e 8,28 g/100 g, para as pataniscas de bacalhau e chamuças de carne, respectivamente. Em 50\% das amostras analisadas, o teor de AGMI foi superior ou igual a $5 \mathrm{~g} / 100 \mathrm{~g}$ de amostra. Os ácidos graxos majoritários em $58 \%$ das amostras analisadas foram poli-insaturados, e os teores destes ácidos graxos variaram entre 3,54 g/100 g (empadas de galinha) e 15,5 g/100 g (chamuças de carne).

Relativamente aos AGT, existe evidência científica que fundamenta a necessidade da redução do seu consumo, sobretudo dos que resultam do uso de óleos vegetais hidrogenados ou parcialmente hidrogenados, devido ao aumento do risco de desenvolvimento de doenças cardiovasculares (EFSA, 2010; WHO, 2013). Para $83 \%$ das amostras analisadas, os teores de AGT determinados variaram entre $7,76 \mathrm{mg} / 100 \mathrm{~g} \mathrm{e} 18,8 \mathrm{mg} / 100 \mathrm{~g}$ de amostra. As empadas de galinha e de requeijão foram as amostras que apresentaram os teores mais elevados de AGT (54,3 \pm 0,19 mg/100 g e $105 \pm 0,62 \mathrm{mg} / 100 \mathrm{~g}$, respectivamente). De acordo com a Organização Mundial de Saúde, a ingestão de AGT deve ser inferior a 1\% do valor energético total. Em Portugal, não existe legislação para o teor deste tipo de ácidos graxos nos alimentos. No entanto, alguns países europeus definem que os teores de AGT devem ser inferiores a $2 \%$ do total de gordura nos alimentos que não contêm gordura láctea ou de ruminantes (WHO, 2015). Nesse sentido, no presente trabalho, verificou-se que todas as amostras de salgados prontos para comer estudados apresentam teores de AGT inferiores a $2 \%$ do teor de gordura total, podendo, deste ponto de vista, ser considerados seguros.

\subsection{Correlação entre teores de gordura, sal e composição em ácidos graxos}

Neste trabalho, as correlações analisadas, de investigação para os diversos parâmetros em estudo, são, em sua maioria, baixas ou moderadas (Tabela 3). No entanto, também se observaram algumas correlações fortes, nomeadamente para os AGS com os AGT $(r=0,81)$ e com os AGMI $(r=0,72)$.

Foi também realizada uma análise da correlação do sal dos salgados com os outros parâmetros em estudo (Figura 2), no sentido de avaliar se os alimentos ricos
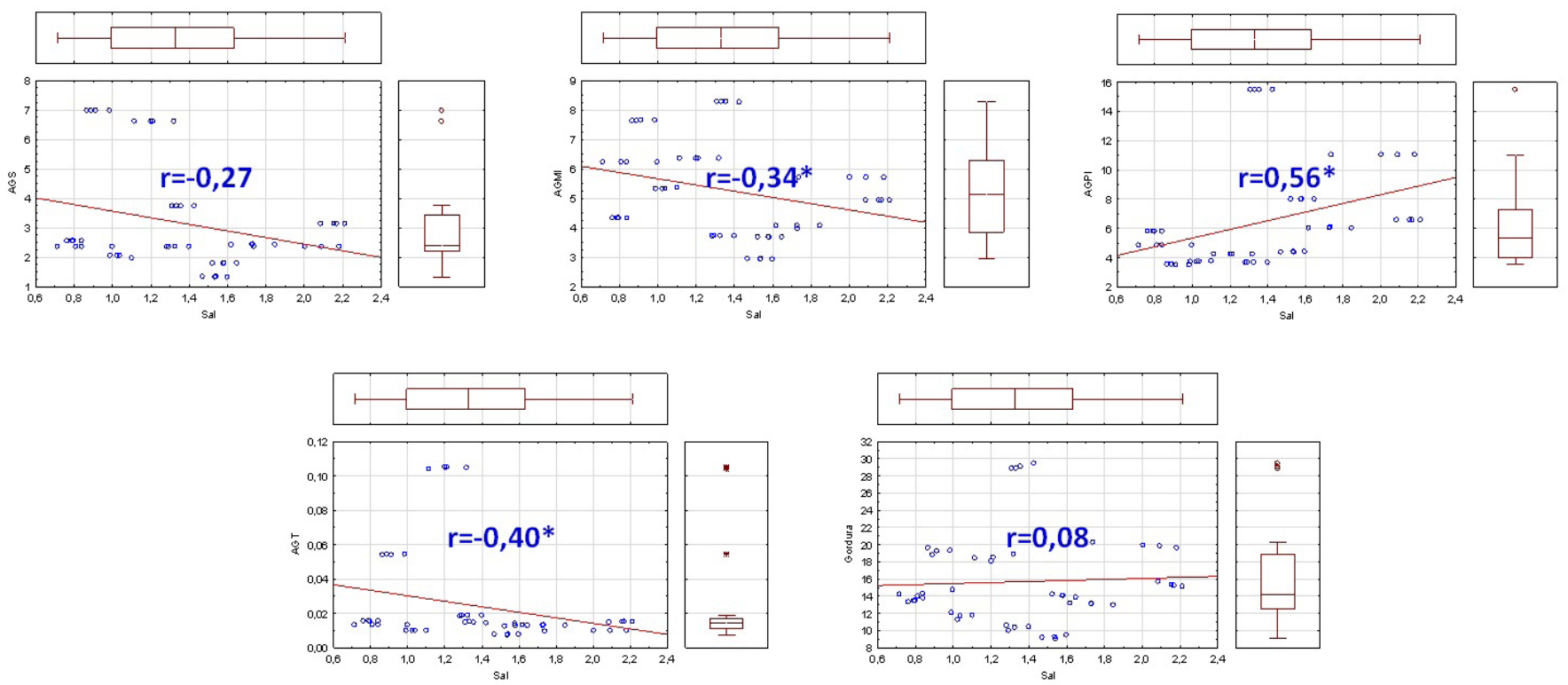

Figura 2. Correlação do teor de sal com os outros parâmetros em estudo ( ${ }^{*}$ diferenças significativas p < 0,05). AGS: Ácidos Graxos Saturados; AGMI: Ácidos Graxos Monoinsaturados; AGPI: Ácidos Graxos Poli-insaturados; AGT: Ácidos Graxos trans. 
Efeito do processamento industrial na qualidade e na segurança de salgados prontos para comer

Albuquerque, T. G. et al.

Tabela 3. Coeficiente de Spearman para as correlações entre os parâmetros analisados.

\begin{tabular}{|c|c|c|c|c|c|c|}
\hline Variáveis & Gordura & Sal & AGS & AGMI & AGPI & AGT \\
\hline Gordura & 1,00 & & & & & \\
\hline Sal & 0,08 & 1,00 & & & & \\
\hline AGS & $0,62^{*}$ & $-0,27$ & 1,00 & & & \\
\hline AGMI & $0,80^{\star}$ & $-0,34^{*}$ & $0,72^{*}$ & 1,00 & & \\
\hline AGPI & $0,45^{\star}$ & $0,56^{*}$ & $-0,10$ & 0,02 & 1,00 & \\
\hline AGT & $0,32^{*}$ & $-0,40^{*}$ & $0,81^{*}$ & $0,42^{*}$ & $-0,35^{\star}$ & 1,00 \\
\hline
\end{tabular}

AGS: Ácidos Graxos Saturados; AGMI: Ácidos Graxos Monoinsaturados; AGPI: Ácidos Graxos Poli-insaturados; AGT: Ácidos Graxos trans; ${ }^{*}$ Diferenças significativas $p<0,05$.

em sal têm, por exemplo, um menor teor de gordura. Verificou-se que a correlação do sal foi mais forte para os ácidos graxos poli-insaturados $(r=0,56)$, ou seja, observou-se uma correlação entre estes dois parâmetros nos salgados. Apesar de as correlações do sal com os outros parâmetros terem sido significativas $(p<0,05)$, exceto para os AGS e a gordura total, estas são fracas ou moderadas, não sendo possível estabelecer uma relação. No entanto, se analisarmos, por exemplo, as chamuças de carne e os croquetes de carne, verifica-se que aquele que tem o maior teor de gordura, tem o menor teor de sal, e vice-versa.

\section{Conclusões}

De acordo com os resultados apresentados, pode afirmar-se que, apesar de as amostras analisadas apresentarem teores de gordura elevados, essa gordura é majoritariamente insaturada. Além disso, os teores de AGT foram inferiores a $2 \%$ do teor total de gordura, indicando que estes alimentos são seguros, no que se refere a este tipo de ácidos graxos. No entanto, deve reiterar-se que, no que diz respeito ao teor de sal, é necessário reforçar a colaboração com a indústria alimentar, no sentido de desenvolver técnicas que permitam a adição controlada de sal, bem como a redução de forma gradual e progressiva do seu teor neste tipo de alimento. Até a data atual, tanto quanto é do conhecimento dos autores, não constava nenhum estudo tão detalhado em relação aos salgados prontos para comer. Desta forma, este trabalho constitui uma atualização de informação no que diz respeito à qualidade nutricional deste tipo de salgados, podendo ser muito útil na avaliação de hábitos alimentares e na estimativa de ingestão de alguns nutrientes relacionados com efeitos benéficos/prejudiciais para a saúde da população.

\section{Agradecimentos}

Trabalho financiado no âmbito do projeto "PTranSALT - Avaliação de ácidos gordos trans, gordura saturada e sal em alimentos processados: estudo do panorama português (2012DAN828)" e no âmbito do financiamento UID/QUI/50006/2013 - POCI/01/0145/ FEDER/007265, com o apoio financeiro da FCT/MEC através de fundos nacionais e cofinanciado pelo FEDER, no âmbito do Acordo de Parceria PT2020. Tânia Gonçalves Albuquerque agradece pela Bolsa de Doutoramento (SFRH/BD/99718/2014) financiada pela Fundação para a Ciência e a Tecnologia (FCT), pelo Fundo Social Europeu (FSE) e pelo Ministério da Educação e Ciência (MEC).

\section{Referências}

ALBUQUERQUE, T. G.; OLIVEIRA, M. B. P. P.; SANCHES-SILVA, A.; BENTO, A. C.; COSTA, H. S. The impact of cooking methods on the nutritional quality and safety of chicken breaded nuggets. Food \& Function, Cambridge, v. 7, n. 6, p. 2736-2746, $2016 \mathrm{a}$. PMid:27213579. http://dx.doi.org/10.1039/C6FO00353B.

ALBUQUERQUE, T. G.; SANCHES-SILVA, A.; SANTOS, L.; COSTA, H. S. An update on potato crisps contents of moisture, fat, salt and fatty acids (including trans-fatty acids) with special emphasis on new oils/fats used for frying. International Journal of Food Sciences and Nutrition, London, v. 63, n. 6, p. 713-717, 2012. PMid:22171702. http://dx.doi.org/10.3109/09637486.2011.644768.

ALBUQUERQUE, T. G.; SANTOS, F.; SANCHES-SILVA, A.; OLIVEIRA, M. B.; BENTO, A. C.; COSTA, H. S. Nutritional and phytochemical composition of Annona cherimola Mill. fruits and by-products: potential health benefits. Food Chemistry, London, v. 193, p. 187-195, 2016b. PMid:26433307. http://dx.doi. org/10.1016/j.foodchem.2014.06.044.

BERBARI, S. A. G.; PRATI, P.; FREITAS, D. G. C.; VICENTE, E.; ORMENESE, R. C. S. C.; FAKHOURI, F. M. Utilização de coberturas comestíveis para redução de absorção de gordura em produtos estruturados pré-fritos congelados de mandioca. Brazilian Journal of Food Technology, Campinas, v. 14, n. 3, p. 172-180, 2011. http://dx.doi.org/10.4260/BJFT2011140300022.

BOER, M.; MCCARTHY, M.; COWAN, C.; RYAN, I. The influence of lifestyle characteristics and beliefs about convenience food on the demand for convenience foods in the Irish market. Food Quality and Preference, Oxford, v. 15, n. 2, p. 155-165, 2004. http://dx.doi.org/10.1016/S0950-3293(03)00054-5.

DAMY, P. C.; JORGE, N. Determinações físico-químicas do óleo de soja e da gordura vegetal hidrogenada durante o processo de fritura descontínua. Brazilian Journal of Food Technology, Campinas, v. 6, n. 2, p. 251-257, 2003. 
Efeito do processamento industrial na qualidade e na segurança de salgados prontos para comer

Albuquerque, T. G. et al.

EUROPEAN FOOD SAFETY AUTHORITY - EFSA. Scientific opinion on dietary reference values for fats, including saturated fatty acids, polyunsaturated fatty acids, monounsaturated fatty acids, trans fatty acids, and cholesterol. EFSA Journal Parma, Parma, v. 8, n. 3, p. 1461, 2010.

IGNÁCIO, A. K. F.; RODRIGUES, J. T. D.; NIIZU, P. Y.; CHANG, Y. K.; STELL, C. J. Efeito da substituição de cloreto de sódio por cloreto de potássio em pão francês. Brazilian Journal of Food Technology, Campinas, v. 16, n. 1, p. 1-11, 2013. http:// dx.doi.org/10.1590/S1981-67232013005000010.

MALACRIDA, C. R.; JORGE, N. Alterações do óleo de soja e da mistura azeite de dendê: óleo de soja em frituras descontínuas de batatas chips. Brazilian Journal of Food Technology, Campinas, v. 6, n. 2, p. 245-249, 2003.

MOZAFFARIAN, D.; KATAN, M. B.; ASCHERIO, A.; STAMPFER, M. J.; WILLETT, W. C. Trans fatty acids and cardiovascular disease. New England Journal of Medicine, Massachusetts, v. 354, n. 15, p. 1601-1613, 2006. http://dx.doi.org/10.1056/NEJMra054035.

PINTO, A. L. D.; MIRANDA, T. L. S.; FERRAZ, V. P.; ATHAYDE, D. D. Determinação e verificação de como a gordura trans é notificada nos rótulos de alimentos, em especial naqueles expressos "0\% gordura trans". Brazilian Journal of Food Technology, Campinas, v. 9, n. e2015043, 2016.

POLÓNIA, J.; MARTINS, L.; PINTO, F.; NAZARÉ, J. Prevalence, awareness, treatment and control of hypertension and salt intake in Portugal: changes over a decade: the PHYSA study. Journal of Hypertension, Philadelphia, v. 32, n. 6, p. 1211-1221, 2014. PMid:24675681. http://dx.doi.org/10.1097/HJH.0000000000000162.

PORTUGAL. Instituto Nacional de Saúde Doutor Ricardo Jorge. Tabela da composição de alimentos. Lisboa: PortFIR, 2015.
Disponível em: <http://portfir.insa.pt/foodcomp/introduction>. Acesso em: 6 fev. 2017.

SANTOS, J. S.; OLIVEIRA, M. B. P. P. Alimentos frescos minimamente processados embalados em atmosfera modificada. Brazilian Journal of Food Technology, Campinas, v. 15, n. 1, p. 1-14, 2012. http://dx.doi.org/10.1590/S1981-67232012000100001.

SOCIEDADE PORTUGUESA DE HIPERTENSÃO - SPH. Fórum do sal: estratégia política até 2020 da Sociedade Portuguesa de Hipertensão na luta contra o excesso de teor de sal nos alimentos. Revista Portuguesa de Hipertensão, Lisboa, n. 51, p. 1-4, 2016.

UNIÃO EUROPEIA. Regulamento (UE) no 1169/2011 do Parlamento Europeu e do Conselho, de 25 de outubro de 2011, relativo à prestação de informação aos consumidores sobre os géneros alimentícios. Official Journal of the European Union, Europe, 22 nov. 2011. Disponível em: <http://eur-lex.europa.eu/legalcontent/PT/TXT/PDF/?uri=CELEX:32011 R1169\&from=pt>. Acesso em: 6 fev. 2017.

WORLD HEALTH ORGANIZATION - WHO. Mapping salt reduction initiatives in the WHO European Region. Copenhagen: WHO Regional Office for Europe, 2013. Disponivel em: <http://www. euro.who.int/_data/assets/pdf_file/0009/186462/Mapping-saltreduction-initiatives-in-the-WHO-European-Region.pdf >. Acesso em: 6 fev. 2017.

WORLD HEALTH ORGANIZATION - WHO. Eliminating trans fats in Europe: a policy brief. Copenhagen: WHO Regional Office for Europe, 2015. Disponível em: <http://www.euro.who. int/__data/assets/pdf_file/0010/288442/Eliminating-trans-fatsin-Europe-A-policy-brief.pdf?ua=1 >. Acesso em: 6 fev. 2017. 